\title{
O CROGODILIANO SUL-AMERICANO Carandaisuchus COMO SINONÍMIA DE Mourasuchus (NETTOSUCHIDAE)
}

\author{
JEAN-CLAUDE BOCQUENTIN* e JONAS PEREIRA DE SOUZA FILHO*
}

\begin{abstract}
THE SOUTH AMERICAN CROCODILIAN CARANDAISUCHUS AS SYNONYMY OF MOURASUCHUS (NETTOSUCHIDAE). New crocodilian skull from the Rio Acre region (Late Miocene-Pliocene) of Brazil provides a basis to establish synonymy between the generic names Mourasuchus, Price, 1964 and Carandaisuchus Gasparini, 1985 the former name is prior to the later, thus, the family Nettosuchidae as here understood includes the species Mourasuchus nativus (Gasparini 1985).

Keywords: South American, Miocene, Crocodylia, synonymy.
\end{abstract}

RESUMO Os restos fósseis apresentados neste trabalho procedem da região do Rio Acre (Mioceno Superior-Plioceno), Brasil. Baseando-se no estudo de um crânio de crocodilfdeo, os autores propõem considerar Carandaisuchus Gasparini 1985 como sinônimo de Mourasuchus Price 1964, de modo que a espécie previamente denominada Carandaisuchus nativus Gasparini pode ser incluída dentro da família Nettosuchidae sob o nome de Mourasuchus nativus (Gasparini 1985).

Palavras-chaves: América do Sul, Mioceno, Crocodylia, sinonfmia.

INTRODUÇÃO O presente trabalho discorre sobre o achado de um crocodiüano de grande porte encontrado nos sedimentos do Mioceno Superior do Estado do Acre, Brasil. Este material, coletado pela equipe de pesquisas paleontológicas da Universidade Federal do Acre em setembro de 1987, foi interpretado à primeira luz como pertencente à espécie "Carandaisuchus" nativus proposta por Gasparini (1985) a partir de um crânio incompleto procedente do "Mesopotamiense" argentino.

Estudos mais detalhados, efetuados no Laboratório de Paleontologia da Universidade e particularmente o achado da mandíbula associada a um dos crânios coletados, levaram os autores a reconsiderar a validade do gênero "Carandaisuchus", pois o material do Acre se encontrava mais completo e permite estabelecer uma sinonímia genérica com Mourasuchus Prince, de criação mais antiga. Até hoje, o gênero Mourasuchus constava de três espécies: $M$. atopus do Mioceno Médio da Colômbia (Langston 1965 e 1966); M. amazonensis do Mioceno Superior do Brasil (Price 1964) e M. arendsí do Mioceno Superior da Venezuela (Bocquentin 1984).

\section{Abreviaturas utilizadas:}

MLP = Museo de La Plata, Argentina

UFAC $=$ Universidade Federal do Acre, Rio Branco, Acre, Brasil.

\section{TAXONOMIA E DESCRIÇÃO}

Ordem CROCODYLIA

Subordem EUSUCHIA

Família NETTOSUCHIDAE

Gênero Mourasuchus Price, 1964

( = Carandaisuchus Gasparini, 1985)

ESPÉCIE-TIPO: M. amazonensis Price, 1964

DIAGNOSE GENERICA (Revisada de Price 1964 e Langston 1966): Crocodiüano de grande porte, rostro achatado e muito largo; tábua craniana proporcionalmente pequena. Bordo orbital proeminente e fenestra laterotemporal muito grande. Mandíbula esguia, com numerosos dentes, excedendo a 40 .

DISTRIBUIÇÃO GEOGRÁFICA E ESTRATIGRÁFICA: Mioceno Médio da Colômbia; Mioceno SuperiorPlioceno do Brasil, Argentina e Venezuela; Pleistocene da Bolívia (Fig. 1)

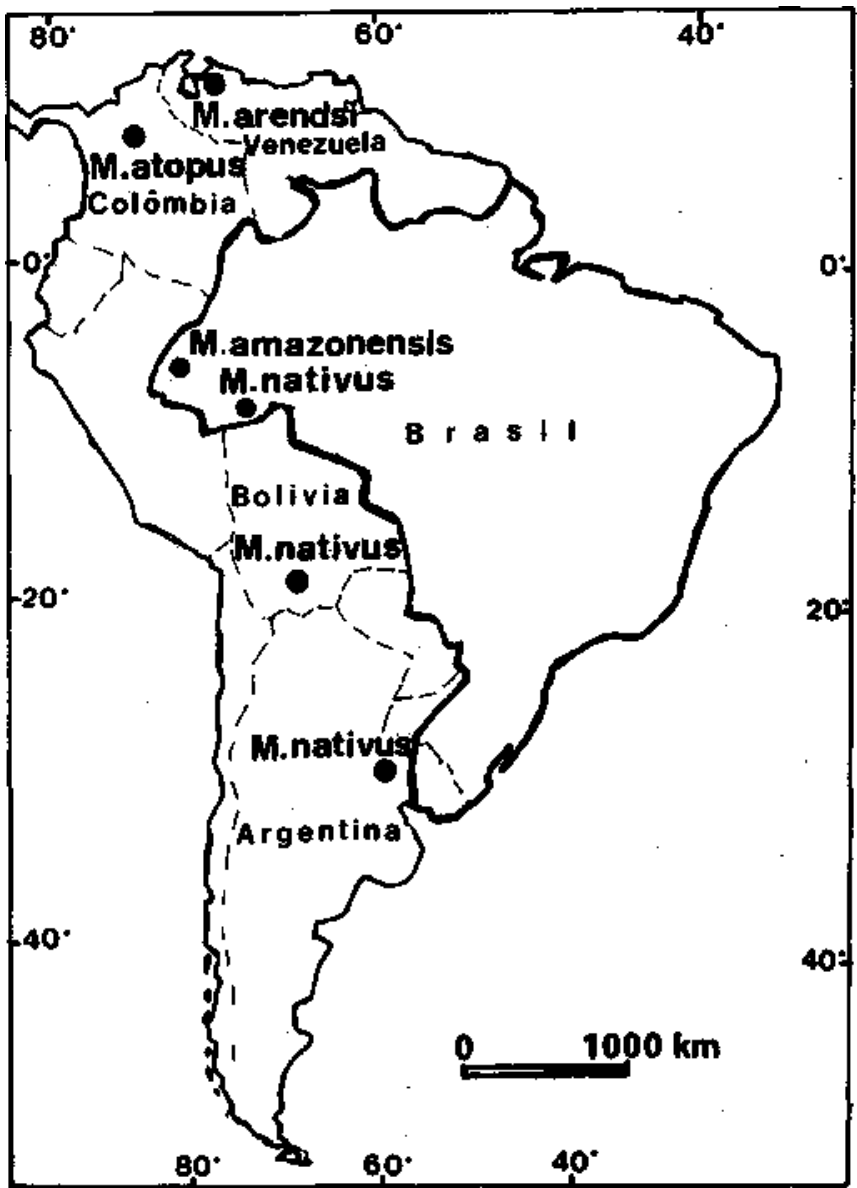

Figura 1 - Mapa de localização

Figure 1 - Locations of the Late Cenozoic sites discussed in the text

Espécie Mourasuchus nativus (Gasparini, 1985)

( = Carandaisuchus nativus Gasparini, 1985)

(Figs. 2, 3 e 4)

HOLÓTIPO: MLP 73-IV-15-8, parte posterior de um crânio

\footnotetext{
* Laboratório de Paleontologia, Departamento de Ciências da Natureza, Universidade Federal do Acre, Campus Universitário, CEP 69900, Rio Branco, AC, Brasil, Bolsista do CNPq
} 
(Gasparini 1985, p. 51).

DISTRIBUIÇÃO GEOGRÁFICA E ESTRATIGRÁFICA: Miocene Superior-Pliocene da Argentina e do Acre, Brasil; e Pleistocene de Tarjja, Bolívia.

DIAGNOSE DIFERENCIAL: $M$. nativus difere de $M$. amazonensis e $M$. arendsi na morfologia da tábua craniana; na linha medial do parietal, localiza-se uma fina crista e toda a metade posterior da tábua é muito elevada. O esquamosal é particularmente alto e forte, configurando uma extraordinária saliência transversal. Em comparação ao M. atopus, difere na disposição mais acentuada do jugal na margem lateral do crânio, à altura das órbitas. Além disso, $M$. nativus apresenta os palatines mais expandidos, tornando mais ampla a distância entre as fenestras palatinas.

Novo material LISTA DO MATERIAL: UFAC 1424, porção posterior de um crânio com ramo mandibular esquerdo associado (Figs. 2A, 3 A e 4); UFAC 1431, porção posterior da tábua craniana e região occipital (Fig. 2B); UFAC 1477; esquamosal direito; UFAC 1495, maxila direita; UFAC 1397, maxila esquerda; UFAC 1485, ramo mandibular direito (Fig. 3B e B'); UFAC 1484, ramo mandibular esquerdo.
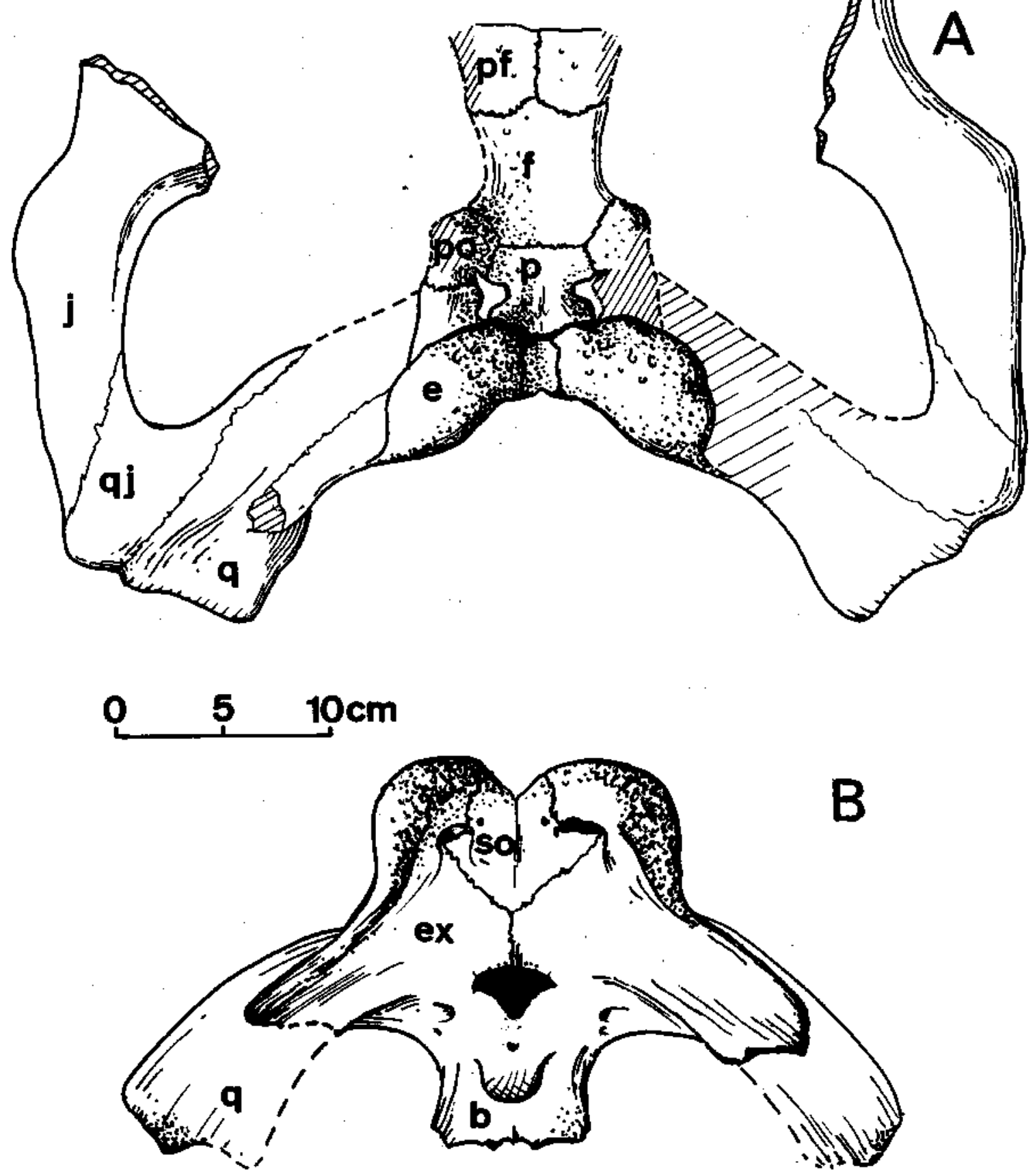

Figura 2 - Mourasuchus nativus (Gasparini 1985). A: UFAC 1424, vista dorsal da porção posterior do crânio - e, esquamosal; f, frontai; j, jugal; p, pariental; pf, pré-frontal; pó, pós-orbital; q, quadrado; q.j, quadradojugal B: UFAC 1431, vista occipital do crânio - b, basioccipital; q, quadrado; só, supraoccipital

Figure 2 - Mourasuchus nativus (Gasparini 1985). A: UFAC 1424, partial skull dorsal view - e, squamosal; f, frontal; j, jugal; p, pariental; pf, préfrontal; pó, postorbital; q, quadrate; qj, quadratojugal. B: UFAC 1431, partial skull occipital view - b. basioccipital; q, quadrate; so, supraoccipital 
PROCEDÊNCIA: Seringal Niterói, num terraço de uma barranca da margem direita do Rio Acre, situada aproximadamente a $20 \mathrm{~km}$ da cidade de Rio Branco, na parte oriental do Estado do Acre, Brasil.

IDADE: Mioceno Superior-Plioceno, ("Edad Huayqueriense-Montehennosense", segundo Mones \& Toledo 1989).

O CRÂNIO: A descrição e a ilustração da região posterior do crânio de "Carandaisuchus" nativus por Gasparini (1985) correspondem totalmente à morfologia observada no material do Acre, até o detalhe tri-radiado das fenestras supratemporais. Ainda assim, a face occipital é alta com as fenestral pós-temporais muito grandes; os esquamosais são hipertrofiados e muito elevados. As fenestras laterotemporais, ausentes no material argentino, presentes no material do Acre, são grandes e semelhantes às de Mourasuchus amazonensis configuradas em Price (1964) e os jugais formam ângulos salientes nas margens laterais do crânio.

Medidas (em mm) do crânio UFAC 1424:

Largura máxima do crânio nas jugais ....

Distância in terorbital. 470

Largura máxima da fenestra palatina

Largura entre as fenestras palatinas

$10 \mathrm{~cm}$
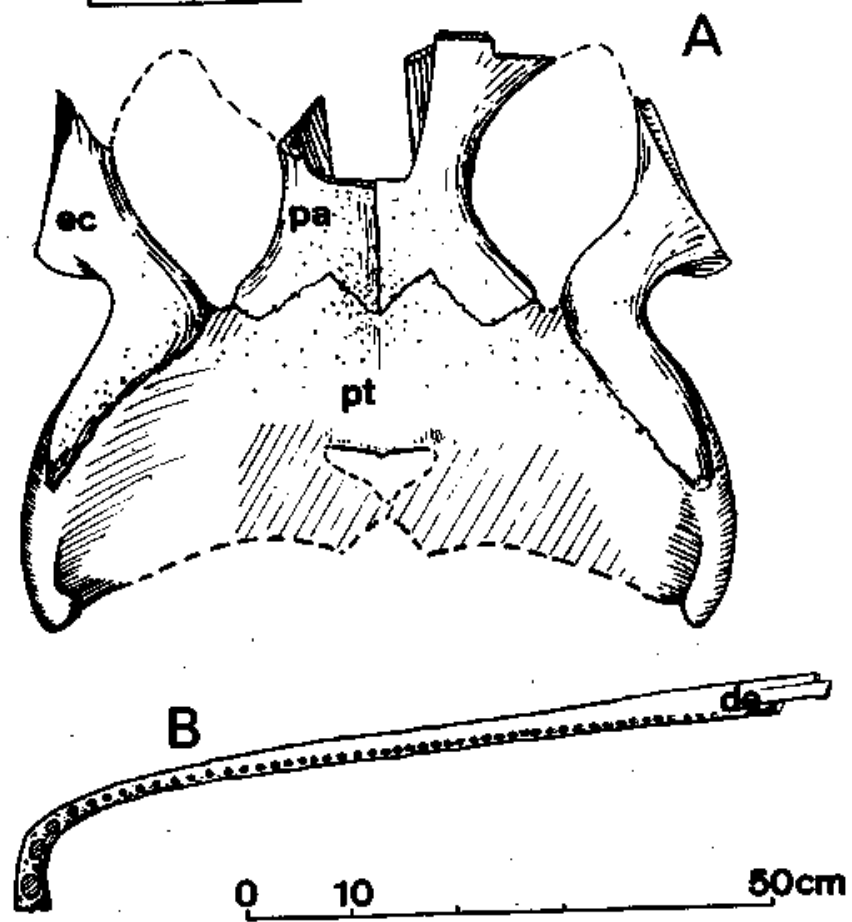

B'

Figura 3 - Mourasuchus nativus (Gasparini 1985). A: UFAC 1424 , vista ventral da região posterior do crânio - ec, ectopterigóide; pá, palatino; pt, pterígóide. $B$ e $B^{\prime}$ : UFAC 1485, ramo mandibular direito incompleto - $B$, vista dorsal: de, dentária; $B^{\prime}$, vista lateral interna: es, esplenial

Figure 3 -Mourasuchus nativus (Gasparini 1985). A: UFAC 1424, partial skull ventral view - ec, ectopterygoid; pa, palatine; pt, pterygoid. B and B': UFAC 1485, partial right mandible B, dorsal view: de, dentaiy; $\mathrm{B}$ ', lingual view: es, splenial

A MANDÍBULA: A mandíbula esquerda (UFAC 1424) foi encontrada articulada ao crânio. Ela consta da maior porção do ramo horizontal até a sínfise e da região articular solta (Fig. 4). A partir das medidas dos fragmentos supracitados pode-se estimar o comprimento total da mandíbula em aproximadamente $1.050 \mathrm{~mm}$. A morfologia geral da mandíbula de M. nativus, até a distribuição dentária, é semelhante à de $M$. atopus descrita e ilustrada por Langston (1965). Não obstante, podemos observar um sulco estendido ao longo da face lateral interna do ramo horizontal das mandíbulas coletadas (Fig. $\left.3 B^{\prime}\right)$. Essa configuração inusitada não tem sido mencionada por nenhum dos autores anteriores.
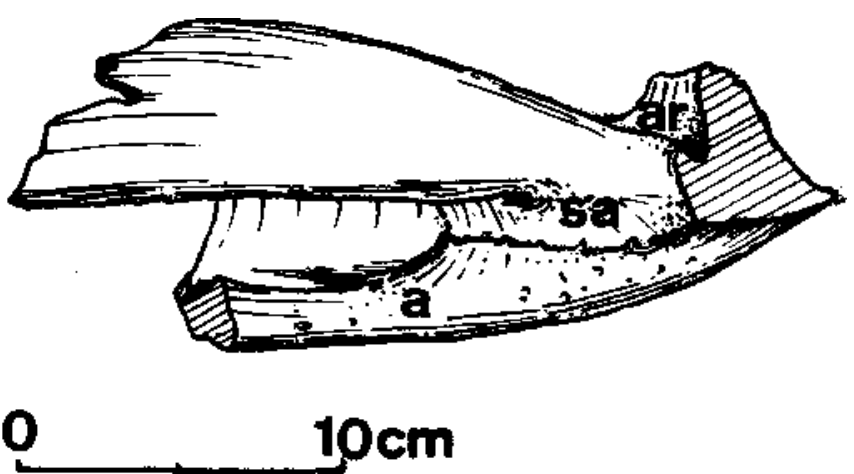

Figura 4 - Mourasuchus nativus (Gasparini 1985). UFAC 1424, vista externa do fragmento relativo à região articular da mandíbula esquerda: a, angular; ar, articular; sa, supra-angular

Figure 4 - Mourasuchus nativus (Gasparini 1985). UFAC 1424, postdental portion of the left mandible: a, angular, ar, articular, sa, suprangular

Considerações taxonômicas Várias características morfológicas que fazem parte da diagnose de Mourasuchus apresentada por Langston (1966) estão também presentes no novo material do Acre que, indubitavelmente, pertence à espécie proposta por Gasparini (1985) e denominada Carandaisuchus nativus. Por dispor de material insuficiente, a autora não pôde afirmar a classificação familiar. Hoje, depois do estudo do Material do Acre, mais completo, não cabe a menor dúvida de que este grande crocodilídeo deva ser classificado na família Nettosuchidae. A tábua craniana, deprimida medianamente, com diminutas fenestras supratemporais, as fenestras laterotemporais grandes e a mandíbula fina e esguia com numerosos dentes, são características de Mourasuchus, único gênero da família. A hipertrofia exagerada dos esquamosais, principal característica considerada pela autora para a proposição da nova espécie $C$. nativus não é totalmente exclusiva dessa espécie. Observa-se, segundo Price (1984), um "engrossamento ósseo" da tábua craniana em M. amazonensis e essa mesma morfologia se verifica também em $M$. arendsi. Assim, acredita-se que essas diferenças no desenvolvimento dos esquamosais sustentam uma distinção específica, mas, por si só, não justificam uma separação genérica. Nessas condições, os autores propõem considerar Carandaisuchus Gasparini, 1985 como sinônimo de Mourasuchus Price, 1964 e Carandaisuchus nativus poderá passar a ser denominada Mourasuchus nativus (Gasparini 1985).

Considerações biogeográficas e estratigráficas Os locais de ocorrência de Mourasuchus no Neógeno da América do Sul estão situados principalmente na área intertropical mas também na região centro-leste da Argentina (ver mapa). Essa distribuição geográfica concorda com os dados fornecidos por Pascual (1984) sobre as deteriorações climáticas ocorridas durante o Terciário e, de um certo modo, confirma a prática 
de considerar os crocodilianos como "termômetros geológicos". O Mioceno Médio, idade da ocorrência de Mourasuchus na Colômbia, é caracterizado, segundo Pascual (1984), por uma fase de diastrofismo intenso que resultou no soerguimento da Cordilheira dos Andes e por um decréscimo dramático das temperaturas mínimas. No entanto, nas altas latitudes, as flutuações das temperaturas foram menores. No mesmo período, uma ampla transgressão submerge a região centroleste do território argentino. A fase seguinte, iniciada por uma regressão marinha, é denominada "idade das planícies australes" por Pascual (1984). Os autores atuais atribuem uma idade Mioceno Superior-Pliocene ("Huayqueriense-Montehermosense") aos sedimentos de origem fluvial ou deltaico do denominado "Mesopotamiense", hoje Formação Ituzaingó da Província de Entre-Ríos, Argentina. Nessa formação foram encontrados os holótípos de $M$. natívus e do grande roedor Euphilus identificado na fauna de Niterói por Mones \& Toledo (1989). O ambiente correspondente é descrito por Bondésio (1985) como de uma região "donde alternam biótopos acuáticos variados con bajas planícies con pastizales, como Ias sabanas arboladas intertropicales". Segundo Pascual (1984, p. 16), essas planícies de idade Huayqueriano ("the late Miocene temperate plains") tiveram uma ampla extensão ao longo das vertentes orientais da cadeia dos Andes até chegar na Bolívia e na região amazônica do Peru. A divulgação por Frailey (1986) da fauna da região do Rio Acre permite verificar que as faunas do Mioceno Superior do Acre e das regiões subtropicais são basicamente semelhantes. $\mathrm{O}$ novo material coletado em Niterói corrobora essas observações.

CONSIDERAÇÕES FINAIS Levando em consideração a morfologia das margens póstero-laterais do crânio e a configuração das fenestras laterotemporais, $M$. nativus mostra maiores afinidades com $M$. amazonensis e $M$. arendsi do que $\operatorname{com} M$, atopus, mais antigo e de tamanho menor.

No Estado do Acre, M. amazonensis só foi registrado, até agora, na região do Rio Juruá enquanto $M$. nativus foi apenas encontrado na localidade Niterói. Essa última localidade, descoberta pela equipe do Laboratório de Paleontologia da UFAC em 1987, tem proporcionado verificar uma fauna abundante e diversificada. Nos restos fósseis fragmentários contidos nos sedimentos argilosos dessa localidade constam, além de M. nativus, Purussaurus, Caiman e Ikanogavialis (até hoje só conhecido do Mioceno Superior da Venezuela). Foi registrada também a presença de uma grande tartaruga pertencente provavelmente a Podocnemis. Os mamíferos são representados, além do roedor Euphilus já mencionado por um Megalonychidae e um Toxodontidae, sendo estes estudados pela equipe do Laboratório. Por outra parte, uma falange assinala a ocorrência, nesse sítio, de grandes aves.

Essa fauna de Niterói confirma a existência de conexões faunísticas no Mioceno Superior-Plioceno entre a região do Rio Acre e aquela de influência do Rio Paraná, na província de Entre-Ríos, Argentina. Sugere também um ambiente com extensas áreas de pastagens (propícias às comunidades de mamíferos herbívoros) e um clima temperado.

Agradecimentos Os autores expressam seus agradecimentos à técnica Tânia Maria C. de Paula, da UFAC, ao Sr. Arito Rosas Júnior, bem como aos estudantes Vladimir, Josecflia, Fátima, Selma e Maria José, do Curso de História, que os acompanharam na coleta do material.

\section{REFERÊNCIAS BIBLIOGRÁFICAS}

BOCQUENTIN, J. 1984. Un nuevo Nettosuchidae (Crocodylia, Eusuchia) proveniente da Ia Formación Urumaco (Mioceno Superior), Venezuela. Ameghiníana, 21(1):3-8.

BONDESIO, P. 1985. Kivutherium scWatoyanei n.sp. (Rodentia, Hidrochoeridae, Cardiatheriinae) de Ia Fonnación Ituzaingó de Ia Província de Entre-Ríos (República Argentina). Ameghiníana, 21:(2-4):275-279.

FRAILEY, C.D. 1986. Late Miocene and Holocene Manuals, exclusive of the Notoungulata of the rio Acre region, western Amazonia. Contributions Science, Nat. Hist. Mus Los Angeles Country, 374:1-46.

GASPARINI, Z. 1985. Un nuevo cocodrilo (Eusuchia) cenozóico de América del Sur. In: Brasil. MME-DNPM, Colet. Trab. Paleont. Brasilia, p. 51-53. (Geologia 27, Paleontologia e Estratígrafia 2).

LANGSTON, W. 1965. Fossil crocodilians from Colombia and the Cenozoic history of the Crocodylia in South America. Univ. Col. Geol.Sci.,52:(l-157).

LANGSTON, W. 1966 Mourasuchus Prince, Nettosuchus langston, and the family Nettosuchidae (Reptilia: Crocodilia). Copeia, 4:882-885.

MONES, A. \& TOLEDO, P.M. de. 1989. Primer hallazgo deEuphilus Ameghino, 1889 (Mammalia: Rodentia: neoepiblemidae) en el Neogeno del Estado do Acre, Brasil. Com. Pia. Mus. Hist. Montevideo, 2l(2):I-15.
PASCUAL, R. 1984. Late Tertiary mammals of southern South America as indicators of climatic deterioration. In: Quaternary of South America and Antartic Peninsula. Com. Invest Cient Província Buenos Aires \& Univ. Nac. Comahue, Neuquén (vol. 2).

PATTERSON, B. 1942. Two Tertiary mammals from northern South America. Amer. Mus. Novit., 1173:1-157.

PRICE, L.1.1964. Sobre o crânio de um grande crocodilfdeo extinto do Alto Rio Juruá, Estado do Acre. An. Acad. Bros. Ciênc., 36(1):59-66.

SILL, W.D. 1970. Nota preliminar sobre un nuevo gavial dei Plioceno de Venezuela y una discusión de los gaviales sud-americanos. Ameghiniana, 7(2):151-159.

MANUSCRITO A575

Recebido em 28 de outubro de 1988

Revisão do autor em 28 de marco de 1989

Revisão aceita em 10 de agosto de 1989 